\title{
Morgagni-Larrey diaphragmatic hernia as cause of intestinal obstruction in a patient with Marfan's syndrome
}

\author{
L. Martínez-Lesquereux ${ }^{1}$, P. Parada-González ${ }^{1}$, F. Macías-García ${ }^{2}$ and A. Beiras-Torrado \\ ${ }^{1}$ Digestive Surgery Department and ${ }^{2}$ Gastroenterology Department. University Hospital of Santiago de Compostela. \\ A Coruña, Spain
}

\section{CLINICAL CASE}

We present the case of a 53-year-old woman with Marfan's syndrome, who suffered abdominal pain, nausea and vomiting. Laboratory test showed only elevation of leucocytosis (13,450 cells). Chest radiograph revealed the existence of a large air bubble at midthoracic level. Computed tomography (Figs. 1 and 2) revealed the existence of a left diaphragmatic defect at the anterior midline, with partial gastric herniation through it and conditioning a high-grade gastric obstruction. Almost the entire colon and multiple ileal segments were also at intrathoracic position. Following the introduction of a nasogastric tube suction the patient improved significantly, so a scheduled laparoscopic surgery was performed: reduction of viscera into the abdominal cavity and repairing of the left anterior hernial orifice with the placement of prostheses.

\section{DISCUSSION}

Congenital diaphragmatic hernia is a rare condition. The so-called hernia of Morgagni (anterior location) is about 3-5\% of the diaphragmatic hernias, being the right side the most frequently affected, as the pericardium protects the left side (1).

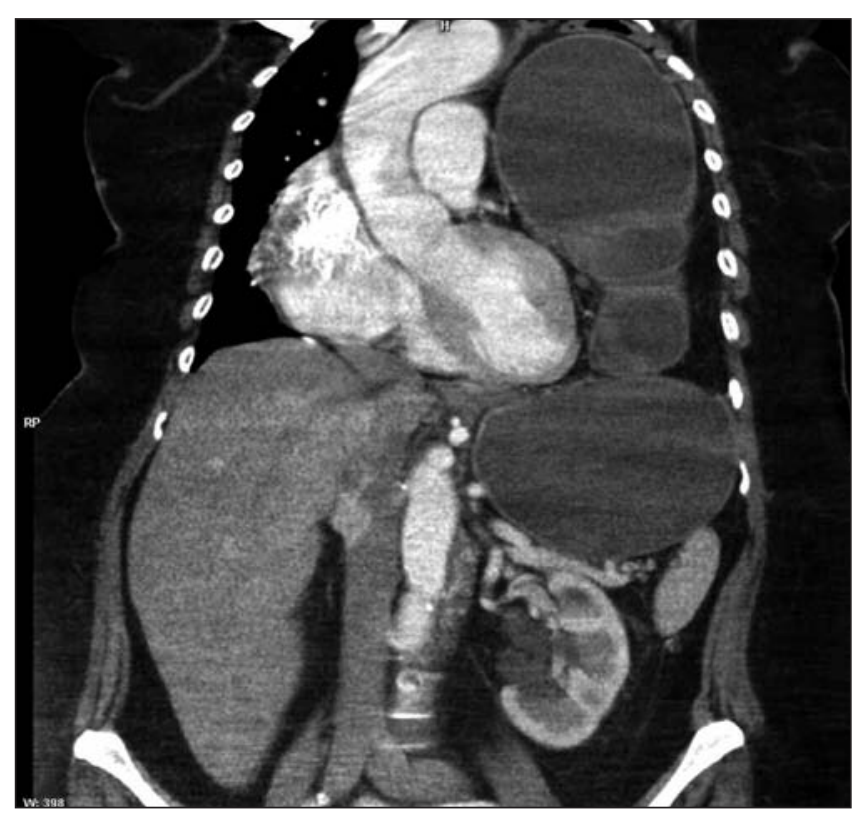

Fig. 1. Thoracoabdominal CT: marked shift back and right of the heart and great vessels secondary to left diaphragmatic hernia.

TC toracoabdominal: marcado desplazamiento posterior y derecho del corazón y grandes vasos secundario a hernia diafragmática izquierda.

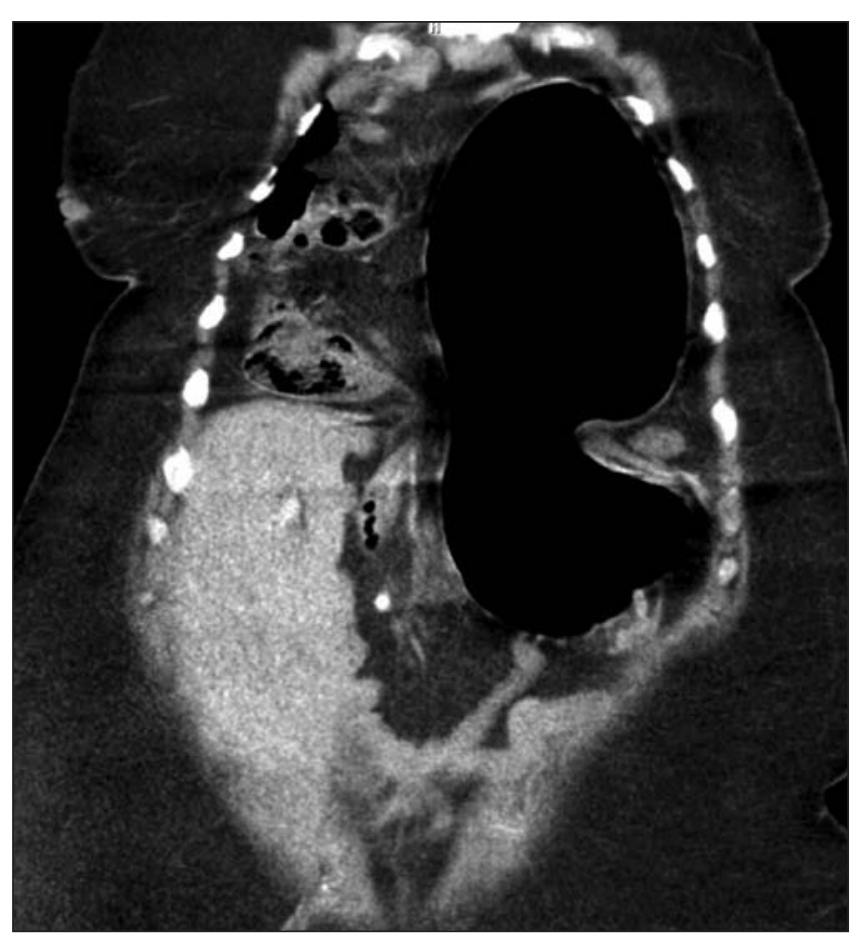

Fig. 2. Abdominal CT: large anterior diaphragmatic defect with herniation of gastric camera, colon and loops of small intestine.

TC abdominal: amplio defecto diafragmático anterior con herniación de cámara gástrica, colon y algún asa de intestino delgado. 
Our case (left anterior congenital diaphragmatic hernia, or Morgagni-Larrey) is, therefore, an entity of exceptional occurrence. The diaphragmatic defect seen in this type of hernia is antero-medially at the junction of the septum transversum of the diaphragm and chest wall (2). The abdominal viscera may not migrate into the chest until late in life, allowing the existence of an asymptomatic diaphragmatic hernia until the development of a complication (3). Surgical treatment is the first option in this pathology, being optional the abdominal or thoracic approach. After reduction of herniated contents into the abdomen, the diaphragmatic defect should be repaired either by primary suture (in cases of small holes) or by the use of a mesh. The laparoscopic approach could be a good option for the management of these patients $(3,4)$.

\title{
REFERENCES
}

1. Arráez-Aybar LA, González-Gómez CC, Torres-García AJ. Hernia diafragmática paraesternal de Morgagni-Larrey en adulto. Rev Esp Enferm Dig 2009; 101(5): 357-66.

2. Stimec BV, Milisavljevic M, Malikovic A, Fasel JH. Omental Morgagni-Larrey hernia: an anatomical pictorial essay. Clin Anat 2008; $21(6): 587-91$.

3. Horton JD, Hofmann LJ, Hetz SP. Presentation and management of Morgagni hernias in adults: a review of 298 cases. Surg Endosc 2008; 22(6): 1413-20.

4. Dapri G, Himpens J, Hainaux B, Roman A, Stevens E, Capelluto E, et al. Surgical technique and complications during laparoscopic repair of diaphragmatic hernias. Hernia 2007; 11(2): 179-83.

\section{Hernia diafragmática de Morgagni-Larrey como causa de obstrucción intestinal en una paciente con síndrome de Marfan}

\author{
L. Martínez Lesquereux ${ }^{1}$, P. Parada González ${ }^{1}$, F. Macías García ${ }^{2}$ y A. Beiras Torrado \\ Servicios de ${ }^{1}$ Cirugía General y del Aparato Digestivo y ${ }^{2}$ Gastroenterología. Hospital Clínico Universitario de Santiago \\ de Compostela
}

\section{CASO CLÍNICO}

Mujer de 53 años con antecedentes de síndrome de Marfan, consultó por cuadro de dolor abdominal, náuseas y vómitos. A la exploración física presentaba disnea y dolor abdominal. Analíticamente destacaba únicamente leucocitosis (13.450 leucocitos) con desviación izquierda. En la radiografía de tórax se evidenció la existencia de una gran burbuja aérea a nivel centrotorácico. La tomografía computerizada (Figs. 1 y 2) mostró la existencia de un defecto diafragmático izquierdo en la región anterior de la línea media a través del cual se herniaba el cuerpo y el antro gástrico, condicionando una obstrucción gástrica de alto grado. La práctica totalidad del marco colónico y varios segmentos de asas ileales se encontraban también en situación intratorácica. Tras la instauración de aspiración por sonda nasogástrica la paciente mejoró significativamente. De forma programada se realizó intervención quirúrgica por vía laparoscópica: reducción de vísceras a la cavidad abdominal y reparación de orificio herniario anterior izquierdo con colocación de prótesis.

\section{DISCUSIÓN}

La hernia diafragmática congénita es una afección infrecuente. La denominada hernia de Morgagni (de localización anterior) supone un 3-5\% del total, siendo el lado derecho el más frecuentemente afectado, pues el pericardio protege el cos- 
tado izquierdo (1). El caso que presentamos (hernia diafragmática congénita anterior izquierda, o de Morgagni-Larrey) es, por lo tanto, una entidad de excepcional incidencia. El defecto diafragmático que se observa en este tipo de hernia es antero-medial en la unión del septum transverso del diafragma y la pared torácica (2). Las vísceras abdominales pueden no migrar al tórax hasta etapas tardías de la vida, posibilitando que la existencia de la hernia diafragmática permanezca inadvertida hasta el desarrollo de alguna complicación (3). El tratamiento de este tipo de patología es quirúrgico, pudiendo optarse por un abordaje abdominal o torácico. Tras la reducción del contenido herniado al abdomen el defecto diafragmático debe repararse, bien mediante sutura primaria (en casos de orificios pequeños) o mediante el uso de mallas. El abordaje laparoscópico es una buena opción para el manejo de estos pacientes $(3,4)$. 